\title{
A Radial Coil Probe for \\ Quadrupole Magnet Measurements
}

\section{Introduction}

This note describes a rotating coil probe of "radial-winding geometry" for the measurements of the magnetic center, quadrupole fields and multipole coefficients of quadrupole magnets. The active length of the coil is longer than the magnet length, so that all the measurements will be integrated values along the longitudinal direction of the magnets. Errors of the measurements due to the fabrication tolerances of the coil are discussed.

\section{Multipole Coefficients}

The magnetic field in the aperture of a magnet may be expressed in the two-dimensional Cartesian or cylindrical coordinate system:

$$
\begin{aligned}
B_{Y}+i B_{X} & =B_{0} \sum_{m=0}^{\infty}\left(b_{m}+i a_{m}\right)(x+i y)^{m}, \\
B_{\theta}+i B_{Y} & =\left(B_{Y}+i B_{X}\right) \exp (i \theta) \\
& =\sum_{n=1}^{\infty} C_{n} \exp \left(-i n \alpha_{n}\right)\left[\frac{z}{R}\right]^{n-1} \exp (i \theta), \\
\text { where } z & =x+i y=r \exp (i \theta),
\end{aligned}
$$

and the multipole coefficients for the two different definitions are related by

$$
\frac{C_{n}}{R^{n-1}} \exp \left(-i n \alpha_{n}\right)=B_{0}\left(b_{n-1}+i a_{n-1}\right) .
$$




\section{DISCLAIMER}

This report was prepared as an account of work sponsored by an agency of the United States Government. Neither the United States Government nor any agency thereof, nor any of their employees, make any warranty, express or implied, or assumes any legal liability or responsibility for the accuracy, completeness, or usefulness of any information, apparatus, product, or process disclosed, or represents that its use would not infringe privately owned rights. Reference herein to any specific commercial product, process, or service by trade name, trademark, manufacturer, or otherwise does not necessarily constitute or imply its endorsement, recommendation, or favoring by the United States Government or any agency thereof. The views and opinions of authors expressed herein do not necessarily state or reflect those of the United States Government or any agency thereof. 


\section{DISCLAIMER}

Portions of this document may be illegible in electronic image products. Images are produced from the best available original document. 
Here $R$ is a reference radius and $b_{1}=1.0 \mathrm{~cm}^{-1}$ for quadrupole magnets. The reference angle for a normal quadrupole magnet is defined as $a_{1}=0$ or $\alpha_{1}=0$. For the storage ring quadrupole $Q 4$ of the APS, $B_{O} b_{1}=C_{2} / R=18.9 \mathrm{~T} / \mathrm{m}$ at $7 \mathrm{GeV}$.

For a quadrupole magnet with effective length $L$, the total flux linkage for coil A at an angular position $\theta$ in Fig. 1 is

$$
\Phi_{A}(\theta)=\sum_{n=1}^{\infty} \operatorname{LN}_{A} C_{n} \frac{1}{n R}\left[r_{A 2}^{n-1}-\left(-x_{A 1}\right)^{n}\right] \cos n\left(\theta-\alpha_{n}\right) .
$$

When the two probe coils, coil A and coil B in Fig. 1, are connected in series such that their flux linkages are opposed to each other, the measurement sensitivity of higher multipoles, $n>2$, could be increased by rejecting the dipole and main (quadrupole) field components of the flux linkages.

The flux linkage for the two coils at $\theta$ is

$$
\phi_{A B}(\theta)=\sum_{n=1}^{\infty} N_{A} C_{n} \frac{1}{n R^{n-1}} I_{A 2}{ }^{n} R_{f a c} \cos n\left(\theta-\alpha_{n}\right),
$$

where

$$
R_{\text {fac }}=1-\left(-r_{A 1} / r_{A 2}\right)^{n}-\left(N_{B} / N_{A}\right)\left[r_{B 2} n-\left(-r_{B 1}\right)^{n}\right] / r_{A 2}{ }^{n}
$$

represents the sensitivity of the measurements for each multipole coefficient. From Eq. (6), the conditions for the rejection of dipole and quadrupole components are given by

$$
\begin{aligned}
& r_{A 2}+r_{A 1}=\left(N_{B} / N_{A}\right)\left(r_{B 2}+r_{B 1}\right), \\
& r_{A 2}-r_{A 1}=r_{B 2}-r_{B 1} .
\end{aligned}
$$


Table 1 lists two examples of the coil locations for $N_{B} / N_{A}=2$ and their $R_{\text {fac. }}$ Each multipole coefficient obtained from the Fourier transformation of the measured data should be divided by the measurement sensitivity, $R_{f a c}$, listed in Table 1 in order to obtain the relative magnitude of the mulfipole coefficient at radius $\mathrm{r}_{\mathrm{Al}}$.

Table 1. Coil Locations and $R_{\text {fac }}$

Parameter

$\mathbf{N}_{B} / N_{A}$

$r_{A 2}$

$\mathbf{r}_{\mathbf{A} \mathbf{1}}$

'B2

$\mathbf{r}_{\mathbf{B} 1}$

n

1

2 (quadrupole)

3

4

5

6

7

8

9

10

11

12

13

14

15
Case \#1

2

$\mathrm{r}_{\mathrm{A} 2}$

$0.5 r_{A 2}$

$0.625 r_{A 2}$

$0.125 r_{A 2}$

$R_{\text {fac }}$

0.000000

0.000000

0.632812

0.632812

0.840454

0.865173

0.933305

0.949527

0.972849

0.980833

0.989119

0.992650

0.995681

0.997163

0.998295
Case \#2

2

$\mathrm{r}_{\mathrm{A} 2}$

$0.55 r_{A 2}$

$0.6125 r_{\text {A2 }}$

$0.1625 r_{A 2}$

$R_{\text {fac }}$

0.000000

0.000000

0.698226

0.628403

0.877692

0.866755

0.950537

0.952010

0.980340

0.982604

0.992289

0.993658

0.997006

0.997676

0.998846 
Magnetic Center and Quadrupole Field Integral

Figure 2 shows two coordinate systems, the $x y$-coordinate system with its origin at the magnetic center (MC) and the 'x' $y$ ' at the cylinder rotation axis (CR). The location of the $C R$ with respect to the $M C$ is $Z_{0}=$ $r_{0} \exp \left(i \theta_{0}\right)$. If the $x^{\prime} y^{\prime}$-coordinate system is displaced from the $x y-$ coordinate system, the expression of the magnetic field at point, $p$, with respect to the two coordinate systems can be expressed as

$$
\begin{aligned}
\sum_{J=1}^{\infty} C_{J}^{\prime} \exp \left(-i J \alpha_{J}^{\prime}\right)\left(\frac{z^{\prime}}{R}\right)^{J-1}=\sum_{n=1}^{\infty} C_{n} \exp \left(-i n \alpha_{n}\right)\left(\frac{z}{R}\right)^{n-1}, \\
\text { where } z^{\prime}=r^{\prime} \exp \left(i \theta^{\prime}\right) \\
z=r \exp (i \theta) \\
=z^{\prime}+z_{0}=z^{\prime}\left(1+z_{0} / z^{\prime}\right) .
\end{aligned}
$$

By using the following relation,

$$
(1+a)^{n-1}=\sum_{k=1}^{n} \frac{(n-1) !}{(k-1) !(n-k) !} a^{k-1}
$$

Eq. (8) becomes

$$
\sum_{J=1}^{\infty} C_{J}^{\prime} \exp \left(i J \alpha_{J}^{\prime}\right)\left(\frac{z^{\prime}}{R}\right)^{J-1}=\sum_{J=1}^{\infty} \sum_{N=J}^{\infty} C_{N} \exp \left(-i N \alpha_{N}\right) \frac{(N-1) !}{(J-1) !(N-J) !}\left(z{ }_{0} / R\right)^{N-J}\left(\frac{z^{\prime}}{R}\right)^{J-1}
$$

Equation (9) shows that the dipole field component in the $x^{\prime} y^{\prime}$ coordinate system consists of the dipole and all other higher multipole components in the xy-coordinate system. If we write up to the sextupole terms of both sides of Eq. (9), we find 


$$
\begin{aligned}
\sum_{J=1}^{3} c_{J}^{\prime} \exp \left(-i J \alpha_{J}^{\prime}\right)\left(z^{\prime} / R\right)^{J-1} & =c_{1} \exp \left(-i \alpha_{1}\right)+c_{2} \exp \left(-i 2 \alpha_{2}\right)\left(z_{0}+z^{\prime}\right)^{/ R} \\
& +c_{3} \exp \left(-i 3 \alpha_{3}\right)\left(z_{0}+z^{\prime}\right)^{2} / R^{2}
\end{aligned}
$$

For a typical quadrupole magnet, $C_{n}(n \neq 2) / C_{2}<1$, the azimuthal field component in the $x^{\prime} y^{\prime}$ - coordinate system due to the main quadrupole field only is

$$
B_{\theta}^{\prime}=\operatorname{Re}\left(C_{2} \exp \left(-i 2 \alpha_{2}+i \theta^{\prime}\right)\left(z_{0}+z^{\prime}\right) ! R\right\} .
$$

The flux linkage for coil A in Fig. 1 is

$\phi_{A}^{\prime}(\theta)=\operatorname{LN}_{A} \frac{C_{2}}{R}\left[r_{0}\left(I_{A 2}+r_{A 1}\right) \cos \left(\theta^{\prime}+\theta_{0}-2 \alpha_{2}\right)+\frac{r_{A 2} 2-r_{A 1}{ }^{2}}{2} \cos 2\left(\theta^{\prime}-\alpha_{2}\right)\right]$.

The off-center of the cylinder rotation axis with respect to the magnetic center can be found from the first term of Eq. (12). The second ferm of Eq. (12) gives the quadrupole field integral, which does not depend on the off-axis distance $z_{0}$. When $z_{o}$ is not zero, it should be noted, however, that there are other correction terms in the quadrupole field integral measurements such as the $2 C_{3} \exp \left(-i 3 \alpha_{3}\right) Z_{0} Z^{\prime} / R^{2}$ term of Eq. (10).

For $L=0.6 \mathrm{~m}, N_{A}=20$ turns, $C_{2} / R=19 \mathrm{~T} / \mathrm{m}, \mathrm{r}_{A 2}+\mathrm{r}_{\mathrm{A} 1}=50 \mathrm{~mm}$ and $\omega=3$ for $\theta=\omega t$, the induced voltage from coil $A$ is

$v_{A}(t)=35(\mathrm{mV}) x_{0}(\mu \mathrm{m}) \sin \left(\omega t+\theta_{0}-2 \alpha_{2}\right)+0.6(\mathrm{~V}) \sin 2\left(\omega t-\alpha_{2}\right)$. 
Equations (12) and (13) indicate that the magnetic center-detecting coil should have radil of $r_{A 2}=r_{A 1}$ to minimize the $\sin (2 \omega t)$ term of the equations.

\section{Coil Position Errors}

In Fig. 3, it is assumed that the plane of coil $A$ is displaced from the cylinder rotation axis by $\Delta r$. When the angular position of $r_{A 2}$ is used as the reference angular position for the measurements, the errors of radial and angular positions of $r_{A 2}$ and $r_{A 1}$ are $\left(\Delta r_{A}, 0\right)$ and $\left(\Delta r_{A}, \Delta \theta\right)$, where $\Delta \theta=\Delta r\left(r_{A 2}+r_{A 1}\right) / r_{A 2} r_{A 1}$. Then the flux linkage of coil $A$ is given by

$$
\phi_{A}(\theta)=\operatorname{LN}_{A}(D+Q) \text {, }
$$

where

$$
\begin{aligned}
D=\frac{C_{2}}{R} r_{0}\left(r_{A 2}+r_{A 1}\right) & {\left[\left(1+\frac{\Delta r+2 \Delta r_{A}}{r_{A 2}+r_{A 1}}\right) \cos \left(\theta^{\prime}+\theta_{0}-2 \alpha_{2}\right)\right.} \\
& \left.+\frac{\Delta r}{r_{A 2}} \sin \left(\theta^{\prime}+\theta_{0}-2 \alpha_{2}\right)\right]_{,} \\
Q=\frac{C_{2}}{2 R}\left(r_{A 2}{ }^{2}-r_{A 1}^{2}\right) & {\left[1+\frac{\Delta r+2 \Delta r_{A}}{r_{A 2}+r_{A 1}}\right.} \\
& \left.+\Delta r \frac{r_{A 1}}{r_{A 2}} \frac{1}{r_{A 2}+r_{A 1}} \sin 2\left(\theta^{\prime}-\alpha_{2}\right)\right] .
\end{aligned}
$$

In Eq. (14) the D and $Q$ terms are used for the measurements of the magnetic center axis and quadrupole field integral, respectively. Relative errors, $\Delta r / r_{A 2}, \Delta r_{2} / r_{A 2}$ and $\Delta r_{1} / r_{A 2}$, in Eq. (14), do not make the procedures for the detection of the magnetic center axis particularly difficult. In the Fourier analysis of the measurement data, however, one should keep in mind the angular phase error due to $\sin \theta^{\prime}$ term. For a relative error of radial positions of $1 \times 10^{-3}$ (for case \# 1 coil geometry in Table 1, $\Delta \theta=3 \times 10^{-3}$ ), the error of the $Q$ term in Eq. (14) would be less than $5 \times 10^{-3}$. 
Relative errors of multiple coefficients due to coil position tolerances for coil A and coil B in Table 1 are listed in Table 2. It is assumed that relative radial position errors of the coils with respect to $\mathrm{r}_{\mathrm{A} 2}$ and relative position errors of the coil planes from its rotation axis are $1 \times 10^{-3}$.

Table 2. Multiple Coefficient Errors

n

1
2
3
4
6
10
Relative errors

$$
\begin{aligned}
& 6 \times 10^{-3} \\
& 7 \times 10^{-3} \\
& 7 \times 10^{-3} \\
& 7 \times 10^{-3} \\
& 9 \times 10^{-3} \\
& 15 \times 10^{-3}
\end{aligned}
$$




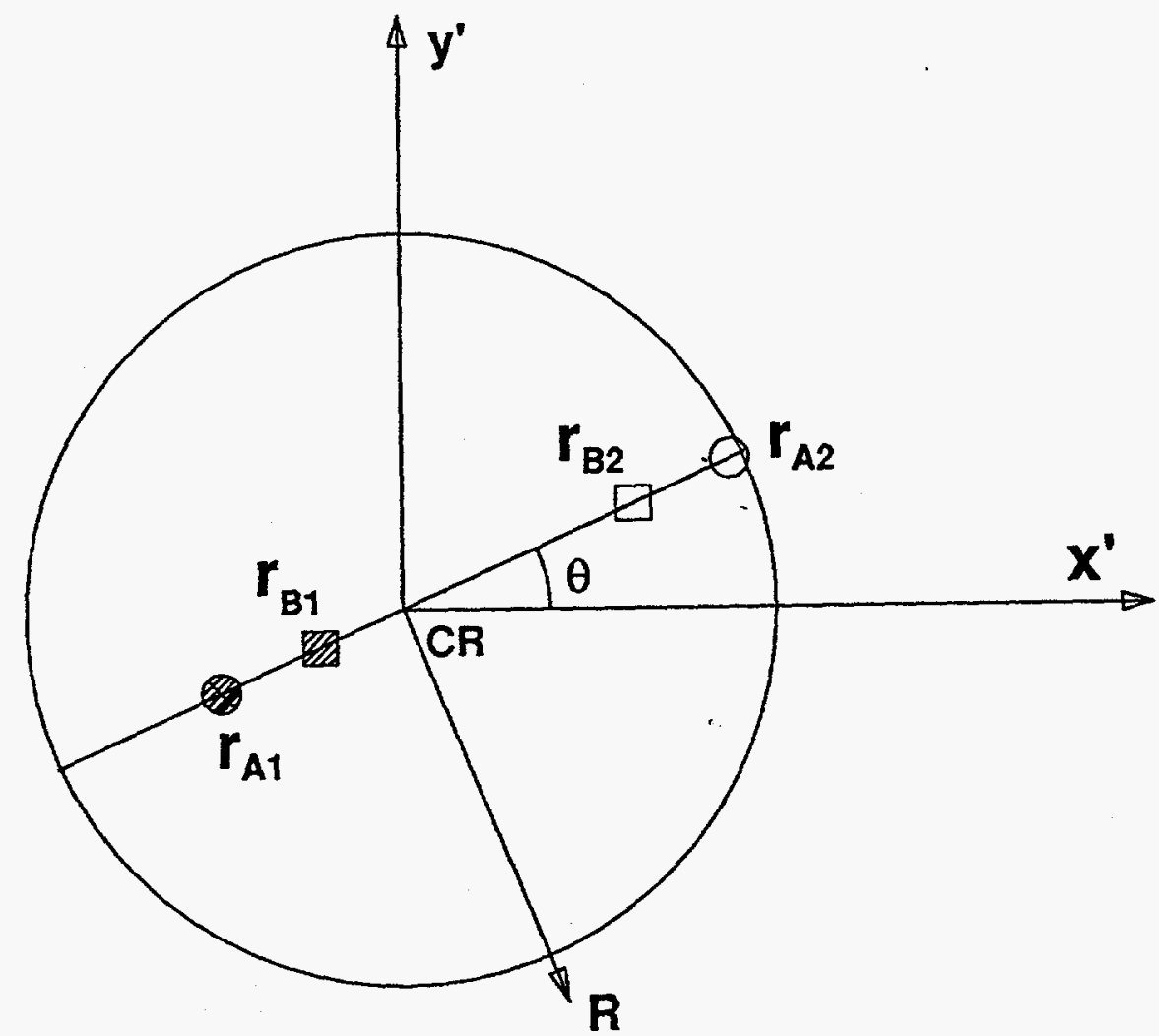

Fig. 1. Cross section of two sets of radial winding coils in a cylinder. Coil $A$ is located at $\left(r_{A 2}, \theta\right)$ and $\left(r_{A 1}, \theta+\pi\right)$, and has a number of turns $N_{A}$. Coil B is located at $\left(r_{B 2}, \theta\right)$ and $\left(r_{B 1}, \theta+\pi\right)$ with $N_{B}$ turns. The cylinder rotation axis $(C R)$ is located at the origin of the $x^{\prime} y^{\prime}$-coordinate system. The $\mathrm{CR}$ is in the plane of the two coils. The radius of the measuring magnet aperture can be chosen as the reference radius $R$. The reference angular position, $\theta=0$, is the direction of the $C R / r_{\mathrm{A} 2}$. 


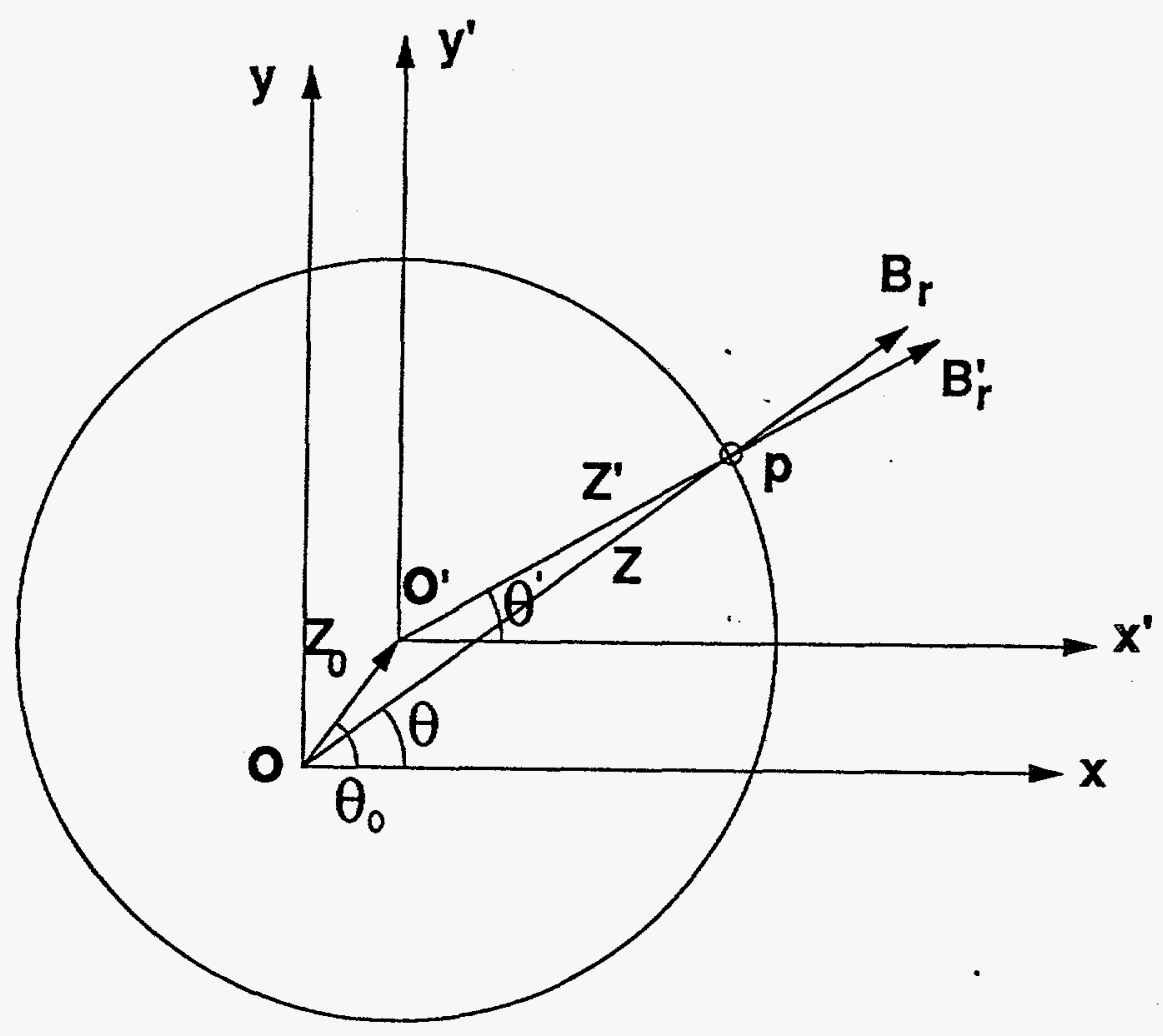

Fig.2. Coordinate systems of the magnetic center (MC) and cylinder rotation axis (CR). The $M C$ is located at $O$. The $C R$, which is located at $O^{\prime}$,is displaced from $M C$ by $Z_{0}=r_{0} \exp \left(i \theta_{0}\right)$.The corresponding axes of the $x y$ and $x^{\prime} y^{\prime}$ coordinates are in parallel. $Z$ and $Z^{\prime}$ are coordinates of point $P$ on the cylinder surface with respect to the two coordinate systems. 


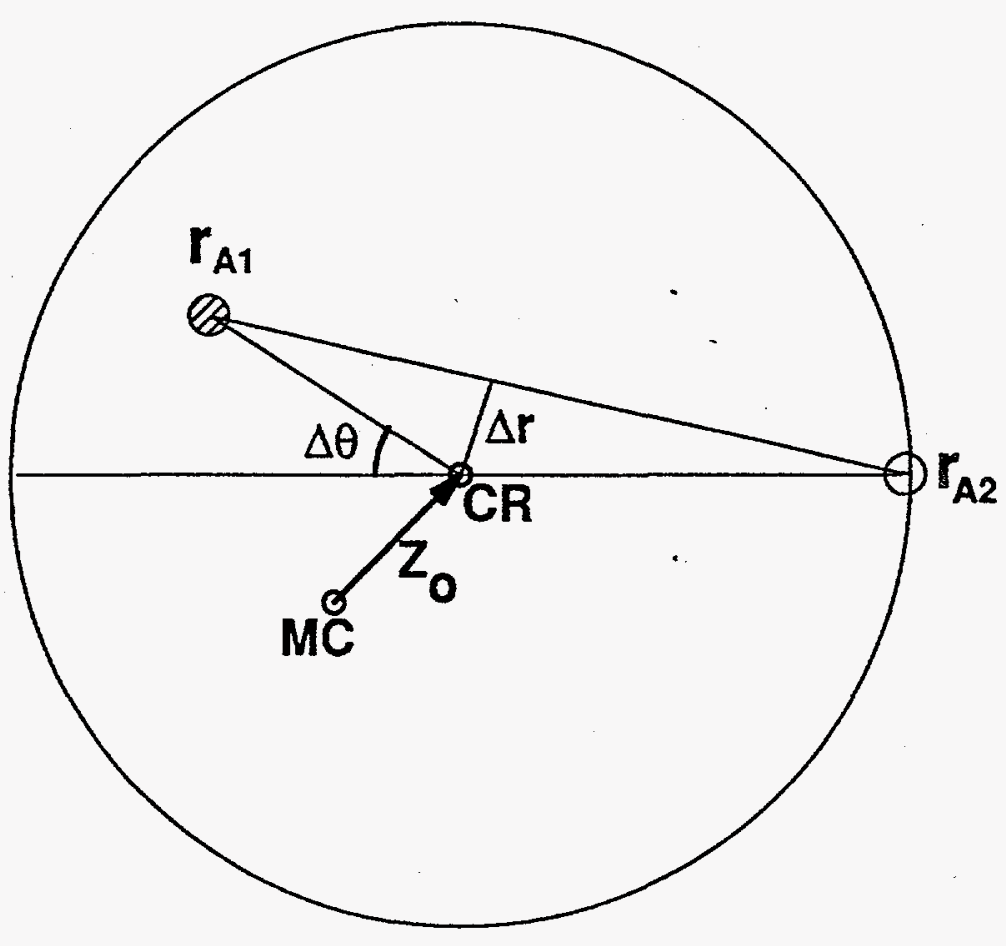

Fig.3. Position error of coil $A$ with respect to the $C R$. The plane of coil $A$ is displaced from the $C R$ by $\Delta r$. Using the angular position of $r_{A 2}$ is used as the reference angular position, $\Delta \theta$ is shown as the angular position error of $r_{A 1}$. 